\title{
Evaluation of Serum Netrin-1 Levels in Psoriasis Patients
}

\author{
Demir Pektas $\mathrm{S}^{1}$, Gokdogan Edgunlu $\mathrm{T}^{2}$ and Karakas Celik $\mathrm{S}^{3}$ \\ ${ }^{1}$ Department of Dermatology, Mugla Sitki Kocman University Faculty of Medicine, \\ Turkey \\ ${ }^{2}$ Department of Medical Biology, Mugla Sitki Kocman University, Turkey \\ ${ }^{3}$ Department of Molecular Biology and Genetic, Bulent Ecevit University, Turkey
}

\section{Research Article \\ Volume 4 Issue 4}

Received Date: December 04, 2019

Published Date: December 31, 2019

DOI: $10.23880 /$ cdoaj- 16000197

*Corresponding author: Suzan Demir Pektas, M.D., Department of Dermatology, Mugla Sitki Kocman University Faculty of Medicine, 48000, Mugla, Turkey, Tel: +90 252211 5219; Fax: +90 312 3116768; Email: suzandpektas@gmail.com

\section{Abstract}

Background: Psoriasis is a chronic, inflammatory and proliferative skin disease, which is associated with comorbidity such as cardiovascular disease and insuline resistance. Vascular alterations observed in psoriatic lesions are now considered to be an important feature of the disease. Netrin-1 is a laminin-related molecule and expressed in tissues such as spinal cord, pancreas, mammary gland, or lung. Netrin-1 has been shown to act as a chemoattractant or chemorepulsive cue for many migrating axons and neurons. The present data published either suggest an antiangiogenic role or a proangiogenic role for netrin-1. A recent study also argues for a role of netrin-1 in autoimmune diseases and in the inflammatory reactions associated to these diseases.

Objective: We aimed to investigate netrin-1 levels in psoriasis patients and its possible relation with duration and severity of disease.

Methods: A total of 40 patients and 40 controls were included in this cross-sectional study, and disease severity was evaluated according to psoriasis area severity index (PASI) scoring. Demographic data, clinical features, anthropometric measures and laboratory findings were recorded in all study subjects. Serum netrin-1 levels were measeured using ELISA kit.

Results: Psoriasis patients had lower values for netrin-1 compared to the control group. But, there were no statistically significant differences in netrin-1 levels between psoriasis patients with PASI>10 and with PASI $\leq 10$. Psoriasis patients with PASI $>10$ had significantly higher PASI, CRP, WBC and RDW values than psoriasis patients with PASI $\leq 10$. We founded that psoriasis patients had netrin was negatively correlated with BMI, duration of disease, PASI and CRP.

Conclusion: Our data shown that there may be a relationship between psoriasis and reduced netrin-1 level. It has been suggested that this relationship may have arisen with chronic inflammation, long disease duration, disease severity and angiogenesis.

Keywords: Psoriasis; Netrin-1; Inflammation; Angiogenesis 


\section{Clinical Dermatology Open Access Journal}

Abbreviations: PASI: Psoriasis Area Severity Index; FPG: Fasting Plasma Glucose; HDL-C: High Density Lipoprotein Cholesterol; LDL-C: Low Density Lipoprotein Cholesterol; DTT: Dithiothreitol.

\section{Introduction}

Psoriasis is immune-mediated chronic and recurrent, inflammatory dermatosis skin disease, which is closely associated with oxidative stres [1]. This disease is recurrent apparition of erythematous plaques often covered by silvery scales. The etiologic and genetic factors which affecting psoriasis are not clear. Trauma, drugs, smoking, emotional stress, alcohol and skin infection are among the factors that affect the clinical development of psoriasis $[1,2]$. According to current understanding of the pathomechanisms integral to the psoriatic process, the skin disorder is characterized by decreased apoptotic cell death of keratinocytes, increased resistance of intralesional keratinocytes to apoptosis, and angiogenesis [1-3].

Complex interaction between cells in the skin and the immune system leads to chronic inflammation of the skin $(3,4)$. The formation and maintenance of psoriatic plaques is due to an imbalance between anti-inflammatory agents that support leukocyte infiltration and proliferation of keratinocytes. The linkages between genetic susceptibility and inflammatory activity in this disease are thought to be effective in many molecules that concern $\mathrm{T}$ cell activation or differentiation [2]. In addition, vascular changes observed in lesions are thought to be an important feature for the disease. In fact, they are a key component not only as a result of the disease, but also because it can promote skin inflammation through recruitment of leukocytes. Increased angiogenesis resulting from psoriatic skin cells, activation of endothelial cells through pro-inflammatory cytokines, thus demonstrates the relationship between the altered epidermis and the immunological component of the disease.

Recently, angiogenesis has become very important in psoriasis. The vascular network found in these lesions has changed considerably, especially in papillary dermis infiltrated by numerous curved and dilated capillaries. Endothelial cells of the vessels constituting vascularization are activated and promote the expression of several adhesion molecules such as ICAM-1, VCAM-1, Thy-1, E- and $\beta$-selectin for leukocyte uptake. Therefore, this pathological angiogenesis is not only a consequence of the disease, but also it causes leukocyte deposition, inflammation and hence the formation of skin lesions. Psoriatic skin cells promote the expansion of the vascular network, particularly by secretion of pro-angiogenic factors such as keratinocytes, VEGF and angiopoietins. Indeed, angiogenesis supported by VEGF secreting keratinocytes leads to skin lesions mimicking inflammation and psoriasis [1-4]. The inflammatory events associated with psoriatic diseases are not limited to the skin, but also are responsible for numerous comorbid conditions including obesity, hypertension, dyslipidemia and diabetes, cardiometabolic disease, stroke, metabolic syndrome, chronic kidney disease, malignancy [5-7].

Netrin-1 is a laminin-related molecule that was discovered as a diffusible molecule produced by the ventral structure in the initially developing spinal cord. Netrin-1 is a member of the family of homologous molecules such as netrin-3, netrin-G1, netrin-G2 and netrin- 4 / b-netrin $[8,9]$. In recent data, netrin- 1 and its receptors are also expressed in nonneural tissues such as pancreas [9,10], mammary gland [11], or lung [12] that has suggested their role in the morphogenesis of 'branched' organs. Netrin-1 was shown to act as a chemoattractive or chemorepulsive cue for many migrating axons and neurons. This effect is believed to occur through the binding to two main families of type 1 transmembrane receptors: DCC (for deleted in colorectal cancer) and its homolog neogenin and the UNC5H $[9,11]$. Furthermore, the interaction of netrin-1/receptors and, more specifically, netrin-1/UNC5H2, was recently shown to be involved in the morphogenesis of endothelial vessels. Indeed, although it is clearly shown that UNC5H2 expressed by the tip cells is a key player in developmental angiogenesis [12], the present data published either suggest an antiangiogenic role or a proangiogenic role for netrin-1 [13-15]. Some recent studies have shown that netrin- 1 plays an active role in autoimmune diseases and inflammatory reactions associated with these diseases [16-19].

There have been no previous studies of the potential roles of netrin-1 in psoriasis. Based on the aforementioned literature on the roles of inflammation in the pathophysiology of psoriasis, this study suggests that netrin-1 levels might be linked to psoriasis. We tried to to find out if there is any change in serum netrin-1 levels in patients with psoriasis, and to determine the relationship between serum netrin-1 with demographic, clinical and laboratory characteristic.

\section{Materials and Methods}

This cross-sectional study was performed in accordance with the guidelines of Helsinki Declaration and it was approved by the local ethical committee. 


\section{Subjects}

This study reviews 40 patients with psoriasis who were admitted to the department of dermatology and 40 healthy volunteers. The patients with psoriasis plaques, the patients who received topical treatment for the last four weeks, the patients who received systemic treatment in the last three months, the patients with concurrent systemic disorders (coronary artery disease, liver failure, renal failure, malignancy etc), the patients who had a habit of smoking and/or alcohol consumption, the patients with pregnancy and the breastfeeding patients were excluded. Special care was exercised to match the study and control groups for age and sex. Demographic features, anthropometric measures and blood pressure values were recorded. Psoriasis duration was obtained by self report of the patients. Age, weight, height, and body mass index [BMI; weight (kilograms)/ height (meters) ${ }^{2}$ ] were evaluated at baseline. Fasting plasma glucose (FPG), CRP and lipid profile [total cholesterol, triglyceride, high density lipoprotein cholesterol (HDL-C), low density lipoprotein cholesterol (LDL-C)] at diagnosis as metabolic analysis were performed. Low density lipoprotein cholesterol (LDL-C) (LDL=total cholesterol- [HDL + (Triglyceride/5)]) were calculated as previously described. Disease severity was assessed by PASI and grouped as A PASI score below or equal 10 was defined as "mild disease" and above 10 was defined as "moderatesevere disease".

\section{Collection of Blood Samples}

Whole blood was taken into commercially available anticoagulant-treated tubes (treated with potassium oxalate/sodium fluoride or EDTA-treated, citratetreated). Cells were centrifuged for 10 minutes at 1,000$2000 \mathrm{xg}$ using a cooled centrifuge to obtain the plasma and kept at $-80^{\circ} \mathrm{C}$ until analysis.

\section{Laboratory Analysis}

Serum CRP levels were measured by turbidimetry $(660 \mathrm{~nm} / 700 \mathrm{~nm})$ with a Cobas 6000 Analyzer (Roche Diagnostics, USA). Results were compared with those obtained using typical immunoturbidometry. FPG was measured with Cobas 6000 Analyzer (Roche Diagnostics, USA) by using the UV hexokinase method. Triglyceride and total cholesterol was determined with the enzymatic colorimetric assay, HDL-C was determined with the homogenous enzymatic colorimetric assay [20]. Serum IMA levels were measured by using colorimetric assay method previously described by Bar-Or, et al. [21]. This colorimetric method is based on biochemical properties of albumin to bind exogenous cobalt. In brief, $200 \mathrm{lL}$ of a subject serum was added to $50 \mathrm{lL}$ of $0.1 \%$ cobalt II chloride $(\mathrm{CoCl} 2,6 \mathrm{H} 2 \mathrm{O})$ (Sigma-Aldrich Chemie GmbH Riedstrasse 2, Steinheim, Germany) followed by mixing and 10 minutes of incubation in the dark at $37 \mathrm{C}$ to allow for cobalt albumin cobalt binding. Then, a total of $50 \mathrm{lL}$ dithiothreitol (DTT) were added as a coloring agent. After 2 minutes of incubation, $1 \mathrm{~mL}$ of 0.9 sodium chloride was added in order to reduce the binding capacity. The blank was prepared similarly with distilled water instead of DTT. The absorbance of samples was measured at $470 \mathrm{~nm}$ using a spectrophotometer (Jenway 6315 UV/visible Scanning Spectrophotometers, United Kingdom). Each sample was measured in duplicate and the mean value was reported. We have measured serum netrin-1level in serum with Human Netrin-1, Ntn1 ELISA kit (YLA1764HU) was assessed by enzyme-linked immunosorbent assay in partipicants with $\mathrm{pg} / \mathrm{ml}$.

\section{Statistical Analysis}

All analyses were performed using SPSS (Statistical Packages for The Social Sciences) software version 19 (SPSS Inc., Chicago, IL, USA). For the categorical variables, chi-square tests were used. The continuous variables were first evaluated for normality of statistical distribution by Shapiro-Wilk tests. The descriptive statistics included mean \pm standard deviation and median (minimum-maximum) for numerical variables and number and percentage for categorical data. Comparison of two groups with respect to numerical variables was carried out using Student's $t$-test when parametric test assumptions were met or Mann-Whitney $U$ test when those assumptions were not met. A Bonferroni correction was used for multiple comparisons and corrected $p$ values were given the symbol $\mathrm{p}^{\prime}$. $\mathrm{P}$ value less than 0.05 were considered as significantly different. The Spearman correlation tests were used to determine the correlations of continuous variables. A $p$ value of $<0.05$ and a confidence interval of $95 \%$ were considered as statistically significant.

\section{Results}

The patient group included 40 psoriasis patients (19 males and 21 females). The control group included 40 age- and sex- matched healthy individuals (22 females and 18 males). The characteristics of the study groups are summarized in Table 1. Psoriasis patients had significantly higher values for BMI $\left(26.9 \mathrm{~kg} / \mathrm{m}^{2}\right.$ vs. 23.4 $\left.\mathrm{kg} / \mathrm{m}^{2}, \mathrm{p}<0.001\right)$ and WHR $(0.8 \mathrm{~kg} / \mathrm{m}$ vs. $0.7 \mathrm{~kg} / \mathrm{m}$, $\mathrm{p}=0.01$, Table 1) compared to the controls. Psoriasis patients had significantly higher mean FPG $(90 \mathrm{mg} / \mathrm{dL}$ vs. $82 \mathrm{mg} / \mathrm{dL}, \mathrm{p}<0.001$, Table 1$)$, CRP $(9.4 \mathrm{mg} / \mathrm{dL}$ vs. 3.3 $\mathrm{mg} / \mathrm{dL}, \mathrm{p}<0.001$, Table 1 ), and triglyceride levels (111 $\mathrm{mg} / \mathrm{dL}$ vs. 88.5, $\mathrm{p}<0.05$, Table 1) compared to the control 


\section{Clinical Dermatology Open Access Journal}

group. When compared to controls, netrin levels $(442.7 \pm 245.8 \mathrm{pg} / \mathrm{ml}$ vs. $655.5 \pm 393.3 \mathrm{pg} / \mathrm{ml}, \mathrm{p}<0.001$, Table 1) were found to be lower in psoriasis patients According to disease severity, psoriasis patients were divided into two groups: 25 psoriasis patients with PASI $\leq 10$ ( 15 females and 10 males), 15 psoriasis patients with PASI $>10$ (6 females and 9 males). Table 2 summarizes the characteristics of the healthy controls, psoriasis patients with PASI $\leq 10$ and PASI $>10$. When compared to healthy controls, psoriasis patients with PASI $\leq 10$ and PASI $>10$ had significantly higher BMI, WHR, CRP and MCV ( $p^{\prime}<0.05$ for all, Table 2). The psoriasis patients with PASI $\leq 10$ and psoriasis patients with PASI $>10$ had significantly lower netrin- 1 values than healthy controls ( $p^{\prime}<0.05$ for all, Table 2). The psoriasis patients with PASI $>10$ had significantly higher PASI, CRP and WBC values than psoriasis patients with PASI $\leq 10\left(p^{\prime}<0.001\right.$ for all, Table 2). There were no statistically significant differences in netrin-1 levels between psoriasis patients with PASI $>10$ and with PASI $\leq 10$ ( $>0.05$ for all, Table 2$)$.

Table 3 shows the correlations among BMI, disease duration, PASI and CRP with netrin-1 parameters in psoriasis patients. We founded that psoriasis patients had netrin was negatively correlated with BMI, duration of disease, PASI and CRP ( $\mathrm{r}-0.284 ; \mathrm{p}<0.05$, Table $3, \mathrm{r}:-0.461$, r:-0.441 and r:-0.424, $\mathrm{p}<0.001$ for all, Table 3).

\begin{tabular}{|c|c|c|c|}
\hline Parameters & $\begin{array}{c}\text { Control Group } \\
\mathrm{N}: 40\end{array}$ & $\begin{array}{c}\text { Psoriasis Group } \\
\text { N: } 40\end{array}$ & $\mathbf{P}$ \\
\hline $\mathbf{F} / \mathbf{M}$ & $22 / 18$ & $21 / 19$ & NS \\
\hline Age (year) & $32.5(20-50)$ & $33.0(20-60)$ & NS \\
\hline Weight (Kg) & $61(51-76)$ & $70(50-103)$ & 0.002 \\
\hline BMI $\left(\mathrm{kg} / \mathrm{m}^{2}\right)$ & $23.4(19.8-25.9)$ & $26.9(19.1-43.1)$ & $<0.001$ \\
\hline WHR & $0.7(0.65-0.96)$ & $0.8(0.6-1.3)$ & 0.001 \\
\hline Duration of disease (years) & & $10(4-15)$ & \\
\hline $\begin{array}{r}\text { PASI } \\
\end{array}$ & & $8.9(4-19)$ & \\
\hline Systolic BP(mmHg) & $120(100-170)$ & $120(100-150)$ & NS \\
\hline Diastolic BP(mmHg) & $80(60-90)$ & $80(60-100)$ & NS \\
\hline CRP(mg/dL) & $3.3(2.2-5.2)$ & $9.4(4.2-18.9)$ & $<0.001$ \\
\hline Hemoglobin (g/dL) & $13.7(12.1-15.1)$ & $13.2(12.3-14.9)$ & NS \\
\hline White blood cell count(cell/L) & $7490(4210-9780)$ & $7435(3920-12000)$ & NS \\
\hline Neutrophilcount (cell/L) & $4613.0 \pm 1223.2$ & $4291.0 \pm 1356.0$ & NS \\
\hline FPG $(\mathrm{mg} / \mathrm{dL})$ & $82(70-105)$ & $90(7.6-109)$ & $<0.001$ \\
\hline $\operatorname{Albumin}(\mathrm{mg} / \mathrm{dL})$ & $4.7(4.2-5)$ & $4.3(3.4-5)$ & 0.001 \\
\hline LDL $(\mathrm{mg} / \mathrm{dL})$ & $95(25-194)$ & $97.5(37.8-179)$ & NS \\
\hline Triglyceride (mg/dL) & $88.5(49-253)$ & $111(51-318)$ & $<0.05$ \\
\hline TC $(\mathrm{mg} / \mathrm{dL})$ & $177(86-286)$ & $172.5(100-272)$ & NS \\
\hline HDL (mg/dL) & $54(35-95)$ & $50.5(20-93)$ & NS \\
\hline Netrin-1 (pg/ml) & $655.5 \pm 393.3$ & $442.7 \pm 245.8$ & $<0.001$ \\
\hline
\end{tabular}

Table 1: Comparison of the clinical characteristics and biochemical parameters of the control and psoriasis patients. Data were presented as mean \pm SD or median (min-max) $\mathrm{N}=$ number of volunteer; $\mathrm{NS}=$ nonsignificant; $\mathrm{BMI}=$ body mass index; WHR=waist to hip ratio; $\mathrm{F} / \mathrm{M}$ females/males; PASI=Psoriasis area severity index; $\mathrm{BP}=$ blood pressure; $\mathrm{FPG}=$ Fasting plasma glucose; $\mathrm{CRP}=\mathrm{C}$-reactive protein; LDL=lowdensity lipoprotein; TC=Total cholesterol; HDL=high density lipoprotein. The p values $<0.05$ were assessed as statistically significant.

\begin{tabular}{|c|c|c|c|c|}
\hline Parameters & $\begin{array}{l}\text { Control } \\
(n=40)\end{array}$ & $\begin{array}{c}\text { Psoriasis patients } \\
\text { with PASI } \leq 10 \\
(n=27)\end{array}$ & $\begin{array}{c}\text { Psoriasis patients with } \\
\text { PASI }>10 \\
(n=13)\end{array}$ & $\begin{array}{c}\text { Psoriasis patients } \\
\text { with PASI } \leq 10 \text { and } \\
\text { PASI }>10 \\
\text { P'-value }\end{array}$ \\
\hline $\mathbf{F} / \mathbf{M}$ & $22 / 18$ & $15 / 10$ & $6 / 9$ & NS \\
\hline Age (year) & $32.5(20-50)$ & $33(20-60)$ & $32(24-39)$ & NS \\
\hline Weight (Kg) & $63.3 \pm 7.7$ & $73.8 \pm 13.2$ & $69.1 \pm 14.2$ & $<0.05 \dagger$ \\
\hline BMI $\left(\mathrm{kg} / \mathrm{m}^{2}\right)$ & $23.4(19.8-25.4)$ & $27.3(19.1-43.1)$ & $25.3(20.8-40.2)$ & $<0.05 \dagger \ddagger$ \\
\hline
\end{tabular}




\begin{tabular}{|c|c|c|c|c|}
\hline WHR & $0.72(0.65-0.96)$ & $0.8(0.6-1)$ & $0.8(0.7-1.3)$ & $<0.05 \dagger \ddagger$ \\
\hline Duration of disease (years) & & $10(4-12)$ & $11(8-15)$ & $\mathrm{NS}$ \\
\hline PASI & & $8.1(4-10)$ & $12.3(10-19)$ & $<0.001 \S$ \\
\hline Systolic BP (mmHg) & $120(100-150)$ & $120(100-150)$ & $120(100-140)$ & NS \\
\hline Diastolic BP (mmHg) & $80(60-90)$ & $80(60-100)$ & $80(70-80)$ & NS \\
\hline CRP (mg/dL) & $3.4 \pm 0.8$ & $8.3 \pm 2.4$ & $13.2 \pm 3.2$ & $<0.05 \dagger \neq \S$ \\
\hline Hemoglobin (g/dL) & $13.4(12.1-15.1)$ & $13.2(12.6-14.7)$ & $13.4(12.1-15.1)$ & NS \\
\hline $\begin{array}{c}\text { White blood cell count } \\
\text { (cell/L) }\end{array}$ & $7457 \pm 1255$ & $8251 \pm 2191$ & $6626 \pm 13.8$ & $\S$ \\
\hline Neutrophil count (cell/L) & $4603 \pm 1209$ & $3990 \pm 1241$ & $4713 \pm 1159$ & NS \\
\hline FPG (mg/dL) & $82(70-105)$ & $91(75-109)$ & $89(7.6-103)$ & NS \\
\hline LDL (mg/dL) & $92.7 \pm 34.7$ & $99.3 \pm 30.8$ & $100.8 \pm 35.7$ & NS \\
\hline Triglyceride (mg/dL) & $88.5(49-253)$ & $116(59-318)$ & $101(51-304)$ & NS \\
\hline TC $(\mathrm{mg} / \mathrm{dL})$ & $177(86-286)$ & $172(116-272)$ & $175(100-247)$ & NS \\
\hline HDL (mg/dL) & $54(35-95)$ & $48(20-92)$ & $61(20-95)$ & NS \\
\hline Netrin-1 (pg/ml) & 470 (343-2039) & $381(315-492)$ & $382(127-887)$ & $<0.05 † \ddagger$ \\
\hline
\end{tabular}

Table 2: Comparision of Biochemical Characteristics in Psoriasis Patients Whose PASI $\leq 10$ and PASI $>10$ with Healthy Controls.

Data were presented as mean $\pm \mathrm{SD}$ or median (min-max).

$\mathrm{N}=$ number of volunteer; $\mathrm{NS}=$ nonsignificant; $\mathrm{F} / \mathrm{M}=$ females/males; $\mathrm{PASI}=$ psoriasis area severity index; $\mathrm{BMI}=$ body mass index;WHR=waist to hip ratio; $\mathrm{BP}=$ blood pressure; $\mathrm{FPG}=$ Fasting plasma glucose; $\mathrm{CRP}=\mathrm{C}$-reactive protein; $\mathrm{LDL}=\mathrm{low}$ density lipoprotein; TC=Total cholesterol; HDL=high density lipoprotein.

The $\mathrm{p}^{\prime}$ values $<0.05$ were assessed as statistically significant.

$* \mathrm{p}<0.05$ was accepted to be statistically significant.

†There was statistical significance between healthy controls and psoriasis patients with PASI $\leq 10(\mathrm{p}<0.05)$.

$\neq$ There was statistical significance between healthy controls and psoriasis patients with PASI $>10(p<0.05)$.

$\S$ There was statistical significance between psoriasis patients with PASI $\leq 10$ and PASI $>10(p<0.05)$.

\begin{tabular}{|c|c|c|c|c|c|c|c|c|}
\hline & \multicolumn{2}{|c|}{ BMI } & \multicolumn{2}{c|}{ Duration of disease } & \multicolumn{2}{c|}{ PASI } & \multicolumn{2}{c|}{ CRP } \\
\hline & $\mathbf{r}$ & $\mathbf{p}$ & $\mathbf{r}$ & $\mathbf{p}$ & $\mathbf{r}$ & $\mathbf{p}$ & $\mathbf{r}$ & $\mathbf{p}$ \\
\hline Netrin-1 & -0.284 & $<\mathbf{0 . 0 5}$ & $-0.461^{* *}$ & $<\mathbf{0 . 0 0 1}$ & $-0.441^{* *}$ & $<\mathbf{0 . 0 0 1}$ & $-0.424^{* *}$ & $<\mathbf{0 . 0 0 1}$ \\
\hline
\end{tabular}

Table 3: Correlation of netrin with characteristics in psoriasis patients

The $\mathrm{p}$ values $<0.05$ were assessed as statistically significant; they were presented in bold.

$\mathrm{BMI}=$ body massindex; PASI=psoriasis area severity index; $\mathrm{CRP}=\mathrm{c}$-reactive protein.

\section{Discussion}

Psoriasis is a chronic, inflammatory and proliferative skin disease, frequently seen with remissions and relapses $[1,2]$. It is a chronic inflammatory disease affecting 2-4\% of the world-population. It is considered that there is a hereditary predisposition to the disease and the onset of disease can be triggered by trauma, infection, medications and stress at any time in life [1-4]. Netrin- 1 is accepted to partipicate a play role in the development and progression of some disease related to inflammation [8-16]. This study aimed to investigate how a netrin-1 level is altered in psoriasis patients and whether netrin-1 levels correlate with the severity and duration of psoriasis. In this study, patients with psoriasis were shown to have significantly lower levels of netrin-1 than healthy controls. There is no significant difference between the netrin- 1 levels of the patients with moderate to severe psoriasis and the patients with mild psoriasis. It was founded that psoriasis patients had netrin-1 levels was negatively correlated with BMI, serum CRP levels, disease duration and PASI.

The complex interaction between the immune system and skin cells causes chronic inflammation of the skin $[1,3,4]$. The formation and maintenance of psoriatic plaques results from an imbalance between antiinflammatory molecules that promote leaking of leukocyte and proliferation of keratinocytes. The relationship between genetic predisposition and inflammatory activity in this disease become more pronounced when the susceptibility locus associated with activation or differentiation of $\mathrm{T}$ cell is identified [1,2]. On the other hand, vascular changes observed in the lesions 
are thought to be an important feature of the disease. In fact, they are a key component not only as a result of the disease, but also it can promote skin inflammation through the proliferation of leukocytes. An increased angiogenesis caused by psoriatic skin cells and activation of endothelial cells through pro-inflammatory cytokines therefore has a significant relationship between the altered epidermis and the immunological component of the disease [1]. Pro-angiogenic factors stimulate endothelial cells, and I first began to release the proteolytic enzymes that break down the extracellular matrix, invasion, migration and proliferation of endothelial cells there, and finally to the differences in a new capillary tube [22]. The effect of netrin-1 on endothelial cell migration has been shown in different studies. Lu et al. They demonstrated the inhibitory effect of netrin-1 on endothelial cell migration using both the Transwell migration system and the wound healing model [13]. Treatment of growing vessels with netrin-1, especially in the end-cell leads to filopodial retraction, endothelial cell migration has been reported to include the role of netrin-1 antiangiogenic $[13,15]$. However, other studies suggest that using similar models, netrin-1 has a positive effect on endothelial cell motions, and the authors suggest that unlike netrin-1, it will have proangiogenic activity $[23,24]$. Since the same endothelial cell lines are cross-checked between different studies, these discrepancies can be caused by two reasons:

1 . The use of different netrin-1 groups, but more likely cell treatment at different netrin concentrations.

2. Generally, the netrin-1 physiological concentration between assays is $50 \mathrm{ng}$ to $150 \mathrm{ng} / \mathrm{ml}$, since it was first measured in the chicken brain $[9,25]$.

In this way, Wilson and colleagues have performed migratory assays using gradient doses of netrin-1, and observed a maximal activity at $50 \mathrm{ng} / \mathrm{ml}$. On the contrary, there was no significant increase in endothelial cell migration for netrin- 1 concentration superior to $1 \mathrm{ng} / \mathrm{ml}$. Interestingly, this is the minimal dose used in assays performed by Lu et al. meaning that at high doses netrin-1 could lose its ability to induce endothelial cells migration and even prevents endothelial cells movement [13]. Such biphasic "bellshaped" dose-reponse curve has already been observed for other angiogenic molecules such as endostatin, thrombospondin- 1 or angiopoietin and even for PDGF, FGF and VEGF on other cell types, such as mast cells for example [26,27]. Thus, discrepancies observed upon the action of netrin-1 during angiogenesis likely results from the use of rightfully different doses of netrin1. It will be of key importance to determine in which range of concentration netrin-1 exerts its physiological activity in vivo and whether abnormally high levels of netrin-1 could occur under certain pathological conditions. In our study, we found that netrin-1 levels of the patients with psoriasis lower than healthy controls. Morever, netrin-1 correlated negatively with disease severity, disease duration, CRP and BMI in all psoriasis patients.. The decresed netrin possibly reflects the association between inflammation and angiogenesis in psoriasis. When the groups were divided according to the severity of the disease, the netrin levels was not different between mildly severe psoriasis patients and moderatesevere psoriasis patients. Such a contradictory finding of correlation according to other studies may be attributed to the differences in demographic and clinical characteristics of the reviewed patients, variations in biochemical measurement methods and heterogeneity in study populations.

In conclusion, we evaluated netrin-1 levels and their relationship with duration and severity of disease in psoriasis patients in this study. We founded that netrin-1 decreased in psoriasis patients. Cardiovascular risk factors such as higher BMI, dyslipidemia, FPG and CRP values were found to be more common in psoriasis patients. Additionally, psoriasis patients were demonstrated to have a significant negative correlation among netrin-1 levels, disease duration, PASI, CRP and BMI paramaters. Decreased netrin-1 levels may play a role in the pathogenesis of chronic inflammation and increased CVD risk in psoriasis patients. However, these findings should be interpreted carefully as the power of this study is limited by its relatively small cohort and lack of prospective data. Further studies are needed to determine whether netrin levels play a role in the inflammation of psoriasis and its role in metabolic diseases associated with this disease.

Acknowledgements: We are grateful to participants in this study.

Study limitations: This study is limited by its relatively small cohort.

\section{References}

1. Guérard S, Pouliot R (2012) The Role of Angiogenesis in the Pathogenesis of Psoriasis: Mechanisms and Clinical Implications. J Clin Exp Dermatol Res S2: 007.

2. Nestle FO, Kaplan DH, Barker J (2009) Psoriasis. N Engl J Med 361: 496-509. 


\section{Clinical Dermatology Open Access Journal}

3. Bowcock AM, Krueger JG (2005) Getting under the skin: the immunogenetics of psoriasis. Nat Rev Immunol 5: 699-711.

4. Lowes MA, Bowcock AM, Krueger JG (2007) Pathogenesis and therapy of psoriasis. Nature 445: 866-873.

5. Hawkes JE, Chan TC, Krueger JG (2017) Psoriasis Pathogenesis and the Development of Novel, Targeted Immune Therapies. J Allergy Clin Immunol 140(3): 645-653.

6. Takeshita J, Grewal S, Langan SM, Mehta NN, Ogdie A, et al. (2017) Psoriasis and comorbid diseases: Epidemiology. J Am Acad Dermatol 76(3): 377-390.

7. Takeshita J, Grewal S, Langan SM, Mehta NN, Ogdie A, et al. (2017) Psoriasis and comorbid diseases: Implications for management. J Am Acad Dermatol 76(3): 393-403.

8. Mehlen P, Guenebeaud C (2010) Netrin-1 and its dependence receptors as original targets for cancer therapy. Current Opinion in Oncology 22(1): 46-54.

9. Serafini T, Kennedy TE, Galko MJ, Mirzayan C, Jessell TM, et al. (1994) The netrins define a family of axon outgrowth-promoting proteins homologous to C. elegans UNC-6. Cell 78: 409-424.

10. Yebra M, Montgomery AM, Diaferia GR, Kaido T, Silletti S, et al. (2003) Recognition of the neural chemoattractant netrin-1 by integrins alpha6beta4 and alpha3beta1 regulates epithelial cell adhesion and migration. Dev Cell 5(5): 695-707.

11. Jiang Y, Min-tsai L, Gershon M (2003) Netrins and DCC in the guidance of migrating neural crest-derived cells in the developing bowel and pancreas. Development 258(2): 364-384.

12. Srinivasan K, Strickland P, Valdes A, Shin GC, Hinck L (2003) Netrin-1/neogenin interaction stabilizes multipotent progenitor cap cells during mammary gland morphogenesis. Dev Cell 4(3): 371-382.

13. Lu X, Le Noble F, Yuan L, Jiang Q, De Lafarge B, et al. (2004) The netrin receptor UNC5B mediates guidance events controlling morphogenesis of the vascular system. Nature 432(7014): 179-186.

14. Park KW, Crouse D, Lee M, Karnik SK, Sorensen LK, et al. (2004) The axonal attractant Netrin-1 is an angiogenic factor. Proc Natl Acad Sci U S A 101(46): 16210-16215.

15. Larrivee B, Freitas C, Trombe M, Lv X, Delafarge B, et al. (2007) Activation of the UNC5B receptor by Netrin-1 inhibits sprouting angiogenesis. Genes Dev 21(19): 2433-2447.

16. Carvalho AL, Chuang A, Jiang WW, Lee J, Begum S, et al. (2006) Deleted in colorectal cancer is a putative conditional tumor-suppressor gene inactivated by promoter hypermethylation in head and neck squamous cell carcinoma. Cancer Res 66(19): 94019407.

17. Griffiths CE, Barker JN (2007) Pathogenesis and clinical features of psoriasis. Lancet 370(9583): 263271.

18. Barton SP, Abdullah MS, Marks R (1992) Quantification of microvascular changes in the skin in patients with psoriasis. Br J Dermatol 126(6): 569574.

19. Bull RH, Bates DO, Mortimer PS (1992) Intravital video-capillaroscopy for the study of the microcirculation in psoriasis. Br J Dermatol 126(5): 436-445.

20. Friedewald WT, Levy RI, Fredrickson DS (1972) Estimation of the concentration of low-density lipoprotein cholesterol in plasma, without use of the preparative ultracentrifuge. Clin Chem 18(6): 499502.

21. Turell L, Radi R, Alvarez B (2013) The thiol pool in human plasma: the central contribution of albumin to redox processes. Free Radic Biol Med 65: 244-253.

22. Castets $M$, Mehlen $P$ (2010) Netrin-1 role in angiogenesis. Cell Cycle 9(8): 1466-1471.

23. Won Park K, Crouse D, Lee M, Karnik SK, Sorensen LK, et al. (2004) The axonal attractant Netrin-1 is an angiogenic factor. Proc Natl Acad Sci USA 101: 1621016215.

24. Wilson BD, Ii M, Won Park K, Suli A, Sorensen LK, et al. (2006) Netrins promote developmental and therapeutic angiogenesis. Science 2006 313(5787): 640-644.

25. Serafini T, Colamarino SA, Leonardo ED, Wang $\mathrm{H}$, Bedington R, et al. (1996) Netrin-1 is required for 
commissural axon guidance in the developing vertebrate nervous system. Cell 87(6): 1001-1014.

26. Motegi K, Harada K, Pazouki S, Baillie R, Schor AM (2002) Evidence of a bi-phasic effect of thrombospondin-1 on angiogenesis. Histochem J 34(8-9): 411-421.
27. Gruber BL, Marchese MJ, Kew R (1995) Angiogenic factors stimulate mast-cell migration. Blood 1995; 86(7): 2488-2493. 